\title{
Size Effect in Compressive Strength of Grouted Sand - Part II: Modeling of Experimental Data
}

\author{
G. Efremidis ${ }^{1,2}$ and C. Anagnostopoulos ${ }^{3}$ \\ ' Laboratory of Mechanics and Materials, Faculty of Engineering, \\ Aristotle University of Thessaloniki, GR-54124, Thessaloniki, Greece \\ email: efremidi@mom.gen.auth.gr \\ ${ }^{2}$ Sector of Geotechnical Engineering, Department of Civil Engineering \\ University of Thessaly, Pedion Areos, GR-38334 Volos, Greece \\ ${ }^{3}$ Laboratory of Geotechnical Engineering, Faculty of Engineering \\ Aristotle University of Thessaloniki, GR-54124, Thessaloniki, Greece \\ email: ygram@geo.civil.auth.gr
}

\begin{abstract}
The second part of this two-part article, concerning the investigation of size effect phenomena in compressive strength of grouted sand, attempts the modeling of the experimental data coming from a series of laboratory unconfined compression tests on grouted sand specimens. The experiments indicated a pronounced strength reduction as the specimen diameter increases. This is a very valuable observation in cement grouting technique in order to obtain reliable extrapolations of strength values from laboratory specimens to practical geotechnical engineering dimensions. The experimental results are interpreted by means of Carpinteri's Multifractal Scaling Law (MFSL), and Bazant's Size Effect Law, (SEL), since the two models come from very different physical arguments. The fitting of the mean values of the experimental data has been accomplished by using the non-linear least squares method. The results have been described in two different diagrams. The first diagram shows the non-linear regression of the experimental data whereas in the second diagram the bilogarithmic plane is illustrated. The qualitative comparison of the two models provides that both can interpret satisfactory the observed size effect for this specific scale range (1:3). The goodness of fit of the two scaling laws is also addressed in this paper.
\end{abstract}

\section{INTRODUCTION}

The size effect is a subject of increasing interest due to the fact that the current applications in modern technology involve a variety of length scales /1/. Improvement of the engineering properties of soil or rock mass by cement grouting is recognized as a viable soil improvement technique for many applications in 
geotechnical engineering practice. The computational assessment of the functioning or integrity of a geotechnical structure under various load conditions may be impossible or prohibitive because of an excessive computational effort or because of insufficient knowledge of the governing physical process. Furthermore, a full scale specimen, used the same materials as used for the prototype and accounting for the essential conditions is often not realizable because of the cost or hazards $/ 2,3 /$. A geometrically scaled down model experiment may provide substantial information if appropriate similitude laws are observed in the design and performance of this small scale experiment. Then the direct transfer of the experimental results to the full scale situation is possible using the relevant similitude laws as transfer laws. In some cases there is experimental evidence of a size influence on the mechanical behavior of the materials if scaled structures or simple specimens made from the same material are tested. This non-similarity may endanger the validity of the transfer of experimental data from small scale tests to the full scale situation $/ 2$.

In the present paper, a set of experimental data, presented in Part $1 / 4 /$, has been fitted to two scaling laws (MFSL, SEL), 15-11\%. Each model's parameters have been calculated and the comparison between the two different fitting functions has provided us with a means to realize their ability to catch the size effect trend. The purpose of the modeling is to condense and summarize the data by fitting them to a theoretical model that depends on a set of adjustable parameters coming from the underlying theory that the data are supposed to satisfy. Then modeling can be used as a kind of interpolation, where a few data points are extended into a continuous function, in order to make reliable predictions outside the range of experimental results. In Part I, an unconfined compression test on grouted sand specimens was presented, where five groups of different diameter cylindrical specimens were tested. For reasons of geometric similarity the ratio of diameter to length was kept constant and equal to 2 . The results of these tests indicated that the compressive strength decreases with the specimen size towards an asymptotically constant value $/ 4 /$.

\section{THEORETICAL MODELS}

The multifractal scaling law (MFSL), proposed by Carpinteri and his co-workers $/ 5-7 /$, and the size effect law (SEL), proposed by Bazant /8-11/ are used to explain the size effects on the compressive strength of grouted sand specimens. The analytical expression of the MFSL, is the following /5-7/,

$$
\sigma_{u}=f_{t}\left(1+\frac{\ell_{c h}}{d}\right)^{1 / 2}, \text { or } \ln \sigma_{u}=\frac{1}{2} \ln \left[f_{t}^{2}\left(1+\frac{\ell_{c h}}{e^{\ln d}}\right)\right] \text {, }
$$

where: $\sigma_{u}$ is the ultimate tensile strength, $d$ is the specimen size, $f_{t}$ is the lowest nominal tensile strength, and $c_{c h}$ is the characteristic internal length of the material. The two constants $f_{t}$ and $c_{c h}$ will be determined 
by means of a non-linear least-squares numerical algorithm in order to perform the best fitting of the experimental data.

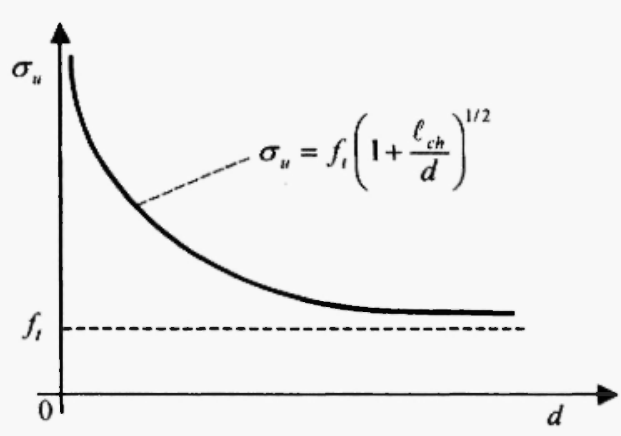

(a)

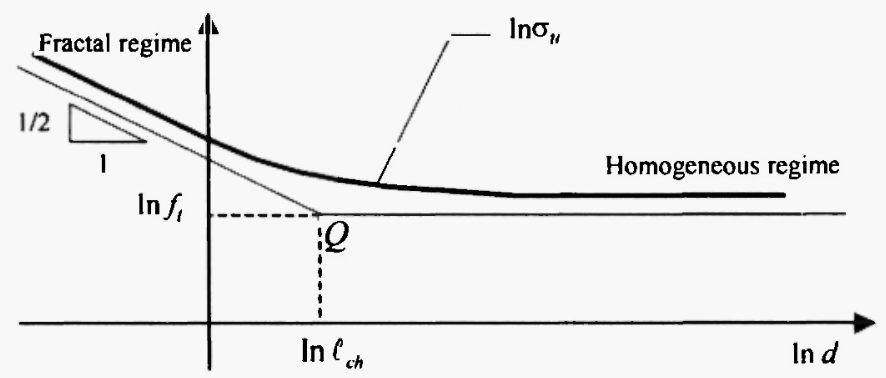

(b)

Fig. 1: Multifractal scaling law (MFSL): (a) proportional diagram, (b) bilogarithmic diagram.

In the proportional diagram of MFSL it is shown that for large specimen sizes the nominal tensile strength $\sigma_{u}$ tends to the asymptotic value $f_{t}$, whereas for small sizes the nominal tensile strength takes distinctly higher values (Fig. Ia). In the bilogarithmic plot (Fig. Ib), the horizontal asymptote corresponds to the large sizes (homogeneous or ordered regime). As the structural size progressively decreases, the mechanical behavior tends to the oblique asymptote (fractal or disorder regime) (Fig. Ib). The intersection point $Q$ of the two asymptotes has horizontal coordinate In $\ell_{c h}$ (Fig. Ib). The microstructural characteristic length $\ell_{c h}$ can be proportional to the maximum size of the smallest particles (grains for metals, crystals for rocks, aggregates for concrete) of the material. In the case of concrete, Carpinteri proposed that $\ell_{c h}=\alpha \cdot d_{\max }$, where $d_{\max }$ is the maximum aggregate size of concrete $16,7 /$. According to MSFL the effect of microstructural behavior becomes progressively less important at larger scales, whereas it represents the fundamental feature at small scales $/ 5 \%$.

The analytical expression of the SEL, where linear elastic fracture mechanics and limit analysis concepts were joined together, is the following $/ 8,9 /$,

$$
\sigma_{u}=\frac{\beta f_{t}}{\sqrt{1+\left(d / d_{0}\right)}} \text {, or } \ln \sigma_{u}=\ln \left(\beta f_{t}\right)-\frac{1}{2} \ln \left(1+\frac{\epsilon^{\ln d}}{d_{0}}\right), \quad / d_{0}=\lambda_{0} d_{\max } /
$$

where: $\sigma_{u}$ is the ultimate tensile strength, $d$ is the characteristic specimen size, $f_{1}$ is strength parameter (it could be the direct tensile strength), $d_{0}$ is an internal length of the material, $\beta, \lambda_{0}$ are two empirical constants determined by fitting test results from geometrically similar specimens of various sizes, and $d_{\max }$ is the maximum aggregate size. It is noted that Eq. 2 was derived initially by Bazant $/ 8,9,11 /$ on the basis of simplified energy release arguments. The ratio $d / d_{0}$ is called the brittleness number $/ 10,11 /$. 


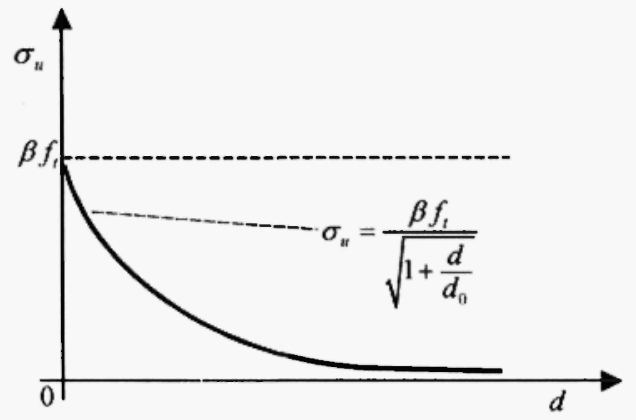

(a)

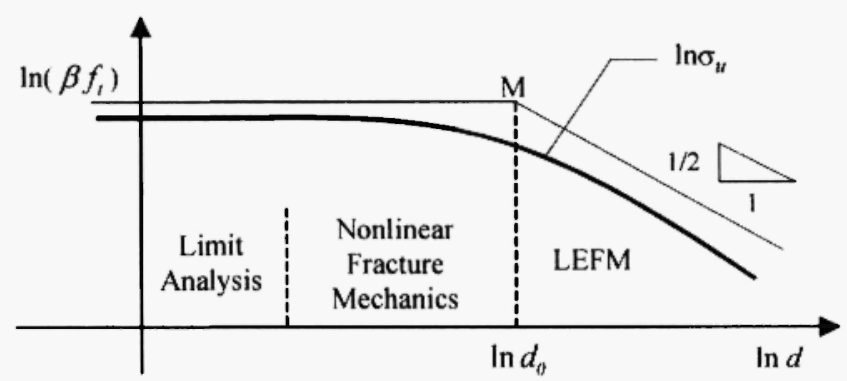

(b)

Fig. 2: Size effect law (SEL): (a) proportional diagram, (b) bilogarithmic diagram.

In the bilogarithmic plot (Fig. 2b), the horizontal asymptote corresponds to the small sizes. As the structural size progressively decreases, the mechanical behavior tends to the horizontal asymptote. This case, where $d / d_{0} \rightarrow 0$ holds, represents a perfectly nonbrittle (plastic ductile) behavior $/ 10,11 /$ (Fig. 2b). For progressively larger structural sizes, the mechanical behavior tends to the oblique asymptote. In this case $\left(d / d_{0} \rightarrow \infty\right)$, where LEFM dominates, a perfectly brittle behavior is represented $/ 10,11 /$. The intersection point $M$ of the two asymptotes has horizontal coordinate $\ln d_{0}$ (Fig. 2b). Because the constant $d_{0}$ depends on structure geometry the definition of brittleness number $d / d_{0}$ is size independent as far as shape independent $/ 11 \%$

\section{FITTING OF THE EXPERIMENTAL DATA}

In Part I of this two-part article, a series of laboratory unconfined compression tests were performed on grouted sand specimens to investigate the size effect phenomena in materials used in cement grouting technique. During these tests, siliceous sand from the Axios river near Thessaloniki was used with maximum grain size $d_{\max }=4.76 \mathrm{~mm}$. During the laboratory experiments 20 permeation groutings were performed on five groups of cylindrical specimens filled with sand including $72 \mathrm{~mm}, 91 \mathrm{~mm}, 122 \mathrm{~mm}, 160 \mathrm{~mm}$ and $200 \mathrm{~mm}$ diameter specimens. The ratio of diameter to length for all specimens was kept constant and equal to 2 in order to retain geometric similarity, $D / L=2 / 4 /$.

From these experiments a pronounced decrease of specimen compressive strength as the specimen diameter increases is observed, i.e. size effect. The fitting of the experimental data has been accomplished by using the non-linear least squares method. The failure mode of compressed grouted sand specimens can be considered as resulting from local tensile mechanisms, or from a combination of tensile and shear mechanisms, according to the specimen geometry. Since the main mechanism in compression is considered 
to be a tensile crack opening, MFSL and SEL can be used to fit the present experimental data and to predict its size effect $/ 12 \%$. The main purposes of the modeling of the present experimental data are: (a) to obtain reliable extrapolations of strength values from laboratory specimens to practical engineering dimensions, and (b) to compare the goodness of fit of MFSL (Eq. 1) with respect to SEL (Eq. 2). In order to quantify the goodness of fit the correlation coefficient $\mathbf{R}$ is used. Comparison of the $\mathbf{R}$ values between the two different fitting functions has provided a mean to test the ability of MFSL and SEL to catch the size effect phenomenon.

Table 1

Grouted Sand Specimens

\begin{tabular}{|c|c|c|c|}
\hline \multirow[b]{2}{*}{ Grouted Sand } & \multicolumn{3}{|c|}{ Compressive strength, $\sigma_{u}(\mathrm{MPa})$} \\
\hline & \multirow{3}{*}{$\begin{array}{c}\text { Experimental } \\
\text { Data } \\
\text { (Average values) } \\
\end{array}$} & \multicolumn{2}{|c|}{ Theoretical Data } \\
\hline & & MFSL & SEL \\
\hline $\begin{array}{l}\text { Diameter, } \\
d(\mathbf{c m})\end{array}$ & & $\begin{array}{c}f_{l}(M F S L)=5.993 \mathrm{MPa}, \\
\ell_{c h}=18.746 \mathrm{~cm}\end{array}$ & $\begin{array}{c}f_{t}(S E L)=15.92 \mathrm{MPa}, \\
d_{0}=6.835 \mathrm{~cm}, \beta=1.01514\end{array}$ \\
\hline 7.2 & 11.84 & 11.38 & 11.28 \\
\hline 9.1 & 10.06 & 10.48 & 10.58 \\
\hline 12.2 & 9.11 & 9.54 & 9.68 \\
\hline 16 & 9.025 & 8.83 & 8.84 \\
\hline 20 & 8.54 & 8.34 & 8.15 \\
\hline
\end{tabular}

For the grouted sand specimens the parameters $f_{t}(M S F L)$ and $\ell_{c h}$ of the MFSL are equal to $5.993 \mathrm{MPa}$ and $187.46 \mathrm{~mm}$, respectively (Table 1). The quantity $\ell_{c h} / d_{\max }$ is equal to 39.38 . The fitting was performed on the averaged values (Fig. 3). The MFSL provides a correlation coefficient $R(M F S L)=0.905$. In case of SEL the parameters $f_{i}(S E L), d_{0}$ and $\beta$ are equal to $15.92 \mathrm{MPa}, 68.35 \mathrm{~mm}$ and 1.01534 , respectively (Table 1). The quantity $\lambda_{0}-d_{0} / d_{\max }$ is equal to 14.36 . The SEL provides a correlation coefficient $R(S E L)=0.842$. The experiments show a $28 \%$ reduction of the compressive strength from a specimen diameter of $7.2 \mathrm{~cm}$ to a specimen diameter of $20 \mathrm{~cm}$ is observed. The use of specimens with diameter of 12.2 $\mathrm{mm}$ or greater indicates mechanical parameters that are reasonable representative the true mass behavior of grouted sand.

For the grout specimens the parameters $f_{t}(M F S L)$ and $\ell_{c h}$ of the MFSL are equal to $6.301 \mathrm{MPa}$ and $201.68 \mathrm{~mm}$, respectively (Table 2). The fitting was also performed on the averaged values (Fig. 4). The MFSL provides a correlation coefficient $R(M F S L)=0.91$. The SEL parameters $f_{l}(S E L), d_{0}$ and $\beta$ are equal to $17.02 \mathrm{MPa}, 62.21 \mathrm{~mm}$ and 1.052 , respectively (Table 2). The SEL provides a correlation coefficient 
$R(S E L)=0.848$. The experimental data present a reduction of $27 \%$ of the compressive strength from a specimen diameter of $7.2 \mathrm{~cm}$ to a specimen diameter of $20 \mathrm{~cm}$.

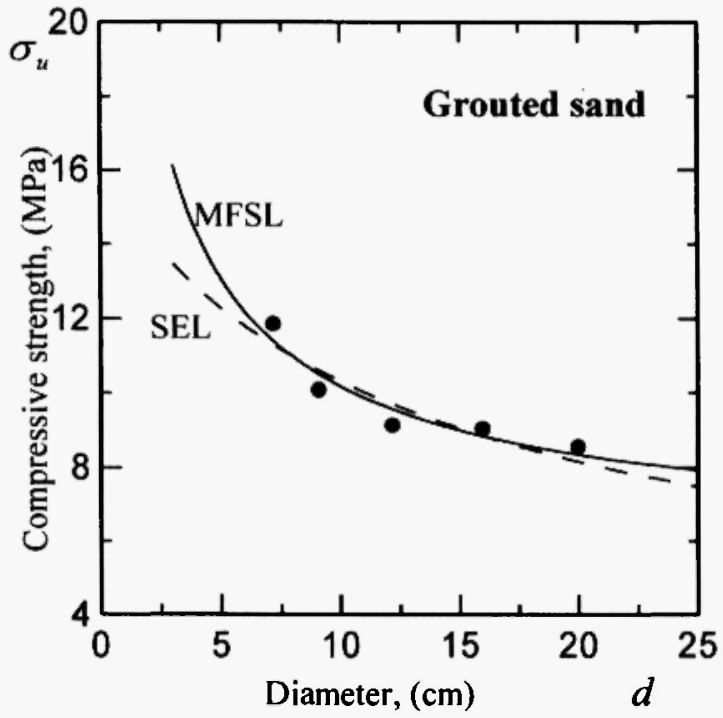

(a)

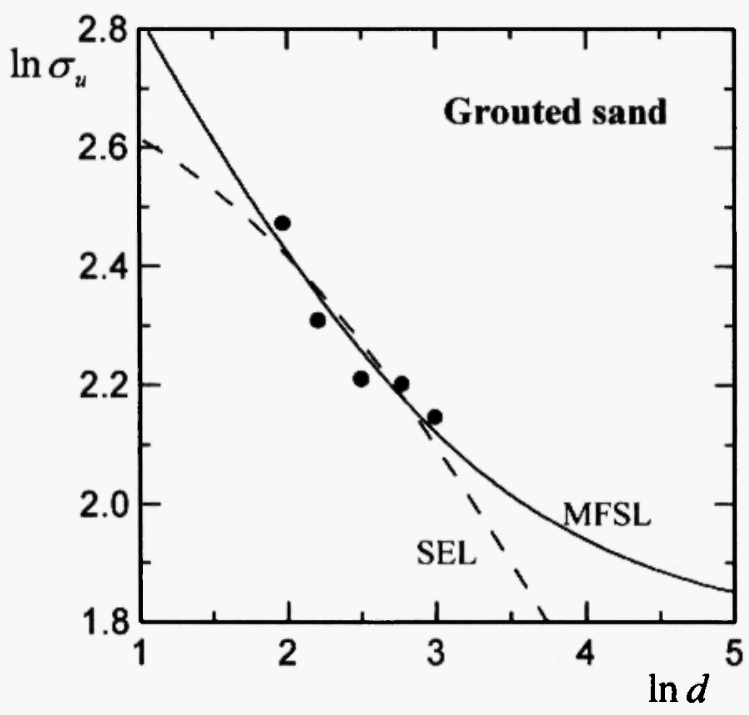

(b)

Fig. 3: Compressive strength data from the tests on grouted sand cylindrical specimens and their fits by the MFSL and SEL: (a) proportional diagram, (b) bilogarithmic diagram.

Table 2

Grout Specimens

\begin{tabular}{|c|c|c|c|}
\hline \multirow[b]{2}{*}{ Grout } & \multicolumn{3}{|c|}{ Compressive strength, $\sigma_{t}(\mathrm{MPa})$} \\
\hline & \multirow{3}{*}{$\begin{array}{c}\text { Experimental } \\
\text { Data } \\
\text { (Average values) }\end{array}$} & \multicolumn{2}{|c|}{ Theoretical Data } \\
\hline & & MFSL & SEL \\
\hline $\begin{array}{c}\text { Diameter, } \\
d(\mathrm{~cm})\end{array}$ & & $\begin{array}{c}f_{t}(M F S L)=6.301 \mathrm{MPa} \\
\ell_{c h}=20.168 \mathrm{~cm}\end{array}$ & $\begin{array}{c}f_{1}(S E L)=17.02 \mathrm{MPa}, \\
d_{0}=6.221 \mathrm{~cm}, \beta=1.052\end{array}$ \\
\hline 7.2 & 12.77 & 12.28 & 12.19 \\
\hline 9.1 & 10.92 & 11.3 & 11.41 \\
\hline 12.2 & 9.76 & 10.26 & 10.41 \\
\hline 16 & 9.55 & 9.47 & 9.48 \\
\hline 20 & 9.25 & 8.93 & 8.72 \\
\hline
\end{tabular}




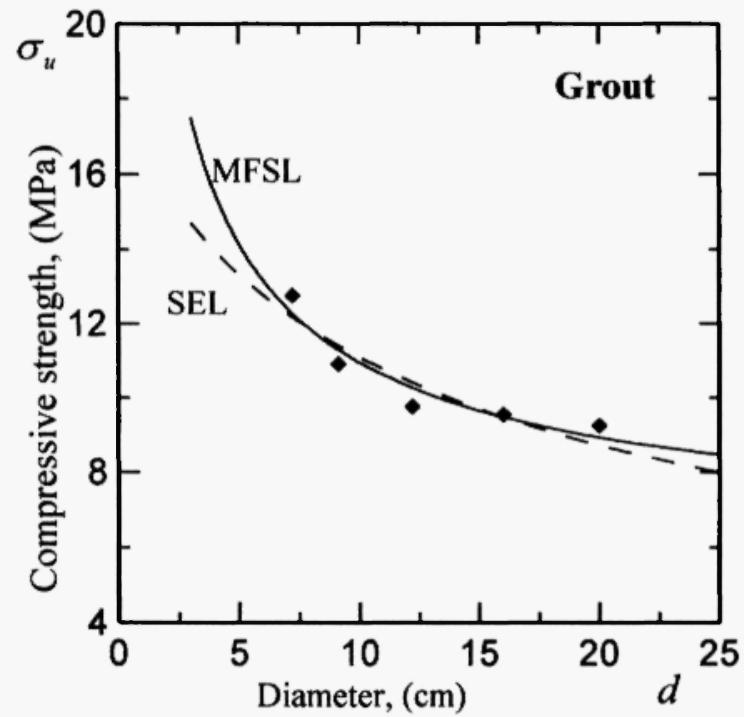

(a)

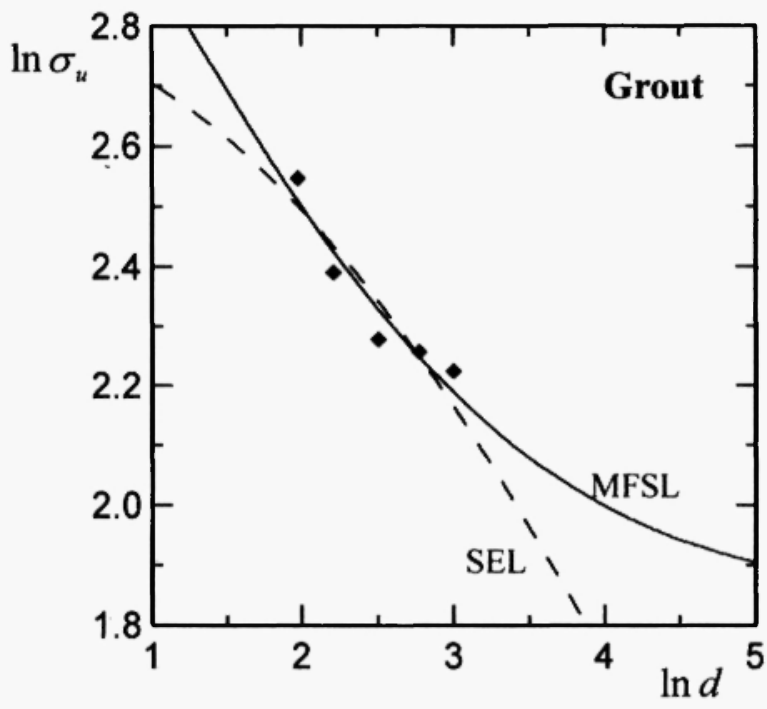

(b)

Fig. 4: Compressive strength data from the tests on grout cylindrical specimens and their fits by the MFSL and SEL: (a) proportional diagram, (b) bilogarithmic diagram.

\section{CONCLUSIONS}

Nowadays the size effect phenomenon is considered as one of the problems of great practical interest and importance in geotechnical engineering due to the fact that its description ensures the validity of the transfer of experimental results from small scale laboratory tests to the full scale situation. In this paper, the multifractal scaling law (MFSL) and the size effect law (SEL) were used to analyze the size effect on a series of experimental unconfined compression tests performed on grouted sand specimens with scale range 1:3. The observed effect of specimen size for diameters of $72 \mathrm{~mm}$ up to $200 \mathrm{~mm}$ shows a strength reduction as the specimen diameter increases. The fitting of the mean values of the experimental data has been accomplished by using the non-linear least squares method. Each model's (MFSL and SEL) parameters have been estimated and the comparison between the two different fitting functions demonstrates their ability to catch the size effect trend, since from the theoretical point of view the two models come from very different physical arguments. The comparison of the goodness of fit between MSFL and SEL was quantified by using correlation coefficient $R$. The calculated values of $R$ for MSFL model are higher that those for SEL. Both models describe satisfactory the size effect trend for the specific scale range though they appear opposite trends in extremely small and large size-scales. 


\section{ACKNOWLEDGEMENTS}

This work was supported in part by the European Community's Human Potential Programme under contract HPRN-CT-2002-00220 (DIGA). We would also like to acknowledge the support of Professor E.C. Aifantis who suggested the use of the MFSL, SEL and his GE (Gradient Elasticity) approach to interpret size effects. The use of the GE approach will be discussed in a forthcoming article by the first author and E.C. Aifantis.

\section{REFERENCES}

1. E.C. Aifantis, Higher order gradients and size effects, In: Size-Scale Effects in the Failure Mechanisms of Materials and Structures, Ed. A. Carpinteri, E \& FN Spon, London 1996; pp. $231-242$.

2 T. Malmberg, I. Tsagrakis, I. Eleftheriadis and E.C. Aifantis, On the gradient plasticity approach to size effects, Part I: Reviews, Forschungszentrum Karlsruhe, FZKA 6321, Germany, 2001.

3. G. Efremidis, "On the theory of gradient elasto-plasticity and scale phenomena", Ph.D. thesis, Aristotle University of Thessaloniki (in Greek), 2002.

4 C. Anagnostopoulos and G. Efremidis, Size effect in compressive strength of grouted sand - Part I: Experiments, Journal of Mechanical Behavior (in print, 2004).

5. A. Carpinteri, Fractal nature of material microstructure and size effects on apparent mechanical properties, Mech. of Materials 18, 89-101 (1994).

6. A. Carpinteri, B. Chiaia and G. Ferro, Multifractal Scaling Law: An extensive application to nominal strength size effect of concrete structures, Report 51, Dept. Struct. Eng. Politech. Turin, Torino, 1995.

7. A. Carpinteri and B. Chiaia, Multifractal scaling laws in the breaking behaviour of disordered materials, Chaos, Solitons \& Fractals 8, 135-150 (1997).

8 Z.P. Bazant, Fracture in concrete and reinforced concrete, in IUTAM Prager Symp on Mechanics of Geomaterials: Rocks, Concretes, Soils, Bazant ZP (ed), NWU, 1983; pp. $281-316$.

9. Z.P. Bažant, Size effect in blunt fracture: Concrete, rock, metal, Journal of Engineering Mechanics 110, $518-535$ (1984).

10. Z.P. Bažant and P.A. Pfeiffer Determination of fracture energy from size effect and brittleness number, ACI Mat. J. 84, 463-480 (1987).

11 Z.P. Bažant and E.-P. Chen, Scaling of structural failure, Appl. Mech. Rev. 50 (10), 593-627 (1997).

12 A. Carpinteri, G. Ferro and I. Monetto, Scale effects in uniaxially compressed concrete specimens, Magazine of Concrete Research 51 (3), 217 - 225 (1999). 